\title{
GlobalizACIÓn, CRECIMIENTO Y DESEMPLEO EN LOS PAÍSES DE LA UE
}

\section{Globalization, Growth and Unemployment in the UE Countries}

Patricia Gómez Costilla Universidad de Valladolid patrygc@fae.uva.es

Julio Herrera Revuelta Universidad de Valladolid jherrera@eco.uva.es

Recibido: abril de 2018; aceptado: octubre de 2018

\section{RESUMEN}

En este trabajo vamos a contrastar empíricamente los efectos de la globalizaciōn sobre el crecimiento y el empleo, puesto que, un gran número de trabajos, desde finales de los años 80 hasta la actualidad, han hecho hincapié en que la globalización ha influido en dichas tasas, aumentando el crecimiento para los ortodoxos, o disminuyéndolo y aumentando la tasa de paro para los poskeynesianos.

Hemos contrastado dichas hipótesis de forma indirecta, por medio de la aplicación de los test de raíz unitaria con perturbaciones en la media. Los resultados confirman que las tasas de crecimiento y de desempleo han sufrido cambios en sus valores de largo plazo, pero han sido otro tipo de perturbaciones, y no la globalizaciōn, los causantes.

Palabras clave: Globalización, crecimiento, desempleo, test de raíces unitarias. 


\begin{abstract}
In this paper we will empirically contrast the effects of globalization on growth and employment, since a large number of works, from the late 1980s to the present, have emphasized that globalization has influenced such rates, increasing growth for the Orthodox, or decreasing it and increasing the rate of unemployment for the post-Keynesians. We have contrasted these hypotheses indirectly, through the application of unit root tests with changes in the mean. The results confirm that the growth and unemployment rates have undergone changes in their long-term values, but there have been other types of disturbances, and not globalization, the causes.
\end{abstract}

Keywords: Globalization, growth, unemployment, unit root test.

Clasificación JEL: F4, E10. 


\section{INTRODUCCIÓN}

Desde los años 90 del siglo pasado existe un gran interés en el estudio sobre los efectos de la globalizaciōn, y más concretamente de la apertura comercial, sobre el crecimiento y el empleo (desempleo), tanto en los países más avanzados, como en el resto del mundo. El aumento del desempleo y la caída del crecimiento económico en esos países ha sido utilizado, por muchos partidos políticos y otro tipo de organizaciones, para trasladar a la opinión pública la idea de que los procesos de apertura e integración económica a nivel mundial son los responsables de esa situación, incluida la crisis financiera. El resultado de esa traslaciōn se observa en el incremento considerable de votos que han tenido partidos de toda índole, cuyo nexo común es la defensa de lo nacional o la reducción de los procesos de integraciōn. A nivel europeo este hecho se ha traducido, no solo en diferentes resultados electorales muy diversos, con un incremento de formaciones que representan la extrema derecha o la aparición en la escena política de partidos nuevos, en los diferentes países, sino también en la celebración de referéndums, como el famoso Brexit o salida del Reino Unido de la UE. 'Esto es la culminación de un proceso que ya empezó a finales de los años 80 con los movimientos antiglobalizadores y las manifestaciones que se celebraban en las reuniones que mantenía la Organización Mundial de Comercio (OMC) en diferentes partes del mundo.

El comercio mundial ha venido evolucionando a lo largo del siglo XX tanto en la cantidad de países, como en la cantidad y variedad de diferentes bienes de exportación e importación intercambiados por cada uno. Podríamos decir que hasta finales de los 80, el comercio mundial de mercancías era básicamente de los países industrializados, fundamentalmente entre ellos, y los países del resto del mundo eran proveedores de materias primas para la producción de dichos bienes $^{2}$. A partir de los 80, por una parte, por la caída del modelo de sustitución de importaciones predominante en la mayoría de los países menos avanzados, y por otra, la entrada en el comercio mundial de, primero los llamados "tigres asiáticos" (Corea del Sur y los pequeños países del sudeste asiático), y, sobre todo, la incorporaciōn de China primero, y el resto de los países de Asia

1 Como el aumento de le extrema derecha en varios países (Austria, Alemania, Grecia, Finlandia, Suecia, Francia) o de partidos antiUE (Podemos en España, Movimiento 5 estrellas en Italia, Syriza en Grecia...).

2 Obviamente esta imagen es una simplificación pero que recoge a grandes rasgos la mayoría del comercio. 
después, ha cambiado cualitativamente ese esquema existente hasta entonces. La presencia de China, sobre todo, ha hecho que parte de la producción y del comercio de mercancías se desplace de los países industrializados tradicionales a esos nuevos países, incluso con la deslocalización de sectores productivos completos debido a los menores costes salariales.

Ese comercio de mercancías con países menos avanzados ha coincidido en el tiempo 3 con un aumento en las tasas de desempleo, y una caída del crecimiento de la producción y la productividad en los países más avanzados. Además, se ha forjado un aumento de la desigualdad en todos los paîses cuya culpabilidad también ha recaído en la globalización. ${ }^{4}$

Para ello, primero haremos un resumen de los resultados empíricos y las predicciones que se pueden concluir de los numerosos trabajos de investigaciōn realizados en los últimos 30 años. Utilizaremos las técnicas de series de tiempo, en concreto los últimos avances econométricos sobre raíces unitarias, refutando la mayoría de las conclusiones obtenidas por dichos autores, para terminar con las preceptivas conclusiones. Para ello hemos seleccionado un grupo numeroso de paîses de la Uniōn Europea (UE) ${ }^{5}$, que poseen todos los ingredientes de la globalizaciōn. ${ }^{6}$ Entre ellos estān países cuya integraciōn ha llegado hasta el nivel de tener una moneda común como es el euro.

Este trabajo trata de aportar algo más de evidencia empírica al debate existente entre la relación de variables como la apertura comercial, el crecimiento y el desempleo, pero de forma indirecta, y no con la tradicional metodología de comparaciōn de países mediante regresiones de corte transversal.

A modo de resumen podemos anticipar que la mayor apertura comercial y el aumento de la integración de las economías en la UE; y concretamente la entrada de países con menores costes salariales en el comercio mundial sí que ha tenido consecuencias sobre el crecimiento y el desempleo en algunos de esos paîses, pero dicha situaciōn ya venía produciéndose antes, con lo que su incidencia no es un hecho irrefutable, sino que sigue abierta al debate.

\section{REVISIÓN DE LAS APORTACIONES DE LOS EFECTOS DE LA GLOBALIZACIŌN Y EL COMERCIO}

La apertura comercial y sus efectos sobre el crecimiento económico y del empleo es un tema, que desde la década de los 80 del siglo pasado, ha tenido una gran repercusión, por lo que las aportaciones de trabajos teóricos y empíricos ha sido muy amplia y variada. Por ello, en este apartado se presenta

\section{Aunque tal coincidencia no es tal como demostraremos más adelante.}

4 Actualmente ha aparecido un nuevo culpable de cara a la opiniōn pública que es el cambio técnico, y otros elementos recurrentes en el tiempo como la inmigraciōn.

5 Dichos países son: Alemania, Austria, Bélgica, Dinamarca, España, Finlandia, Francia, Grecia, Holanda, Italia, Portugal, Reino Unido, Suecia.

6 Tienen diferentes tasas de apertura. Todos son miembros de la UE que es el espacio donde existe libertad de movimiento de mercancías, capital e incluso personas. Además, se han enfrentado a la incorporación de los antiguos países comunistas con unos costes salariales mucho menores (cosa que algunos ya habían hecho antes en sucesivas ampliaciones). 
un análisis desde dos perspectivas: por un lado, se toman en consideración aquellos trabajos cuya línea se establece dentro de un modelo tradicional de comercio, lo que sería el modelo neoclásico; y, por otro lado, se señalan a los autores postkeynesianos. No se pretende hacer un recorrido de todos y cada uno de los análisis que se han realizado desde estas corrientes, pero si se intenta dar una idea clara respecto a las tendencias analíticas que ha habido sobre el tema que nos ocupa.

Existe un primer bloque de trabajos que van desde finales de los 80, y cuyo resumen más conocido es el del artículo de Edwards (1993). En dicho artículo, Edwards se cuestiona la relaciōn empírica obtenida en los trabajos hasta entonces de que la apertura comercial aumente el crecimiento. En dicho resumen, Edwards incluye análisis multipaîs y tambiēn artículos de datos transversales. La mayoría de ellos se centran en la relación entre exportaciones y crecimiento, debido al éxito económico que estaban teniendo los llamados "tigres asiáticos" en la política llamada "crecimiento del lado exportador" (export-led growth). Entre los artículos que Edwards revisa se encuentran los de Feder (1983), Balassa (1985) y Esfahani (1991). La evaluación de Edwards sobre este tema es claramente negativa, donde critica desde la parte empírica de las regresiones cruzadas, como desde el punto de vista teórico de la relaciōn entre las exportaciones y el crecimiento.

Un segundo bloque de trabajos se centra, no en la relación entre el aumento del comercio, sino entre la política comercial y el crecimiento. El artículo de Rodríguez y Rodrik (2001), realiza un resumen de los que considera los principales artículos hasta la fecha en esa área, y, al igual que Edwards, presenta una crítica muy fuerte a las conclusiones y metodología empleada en ellos. Los artículos que Rodríguez y Rodrik revisan son los de Dollar(1992), Sachs y Warner(1995), Ben David(1993) y Edwards (1998), además de Frenkel y Romer(1999), Lee(1993), Harrison (1996), y Wacziarg(1998). Todos ellos concluyen en la importante relaciōn existente entre liberalización comercial y crecimiento económico. Rodríguez y Rodrik, al igual que anteriormente Edwards, se muestran muy críticos con dichos trabajos, es más, utilizando datos actualizados y realizando las mismas regresiones que los trabajos mencionados no llegan a las conclusiones que los autores establecen en ellos.

En resumen, se puede afirmar, que no parece que se pueda demostrar la relaciōn entre la apertura comercial y crecimiento, tanto de la producción como de la productividad. Dicha relación si existe, puede ser cualquiera por lo que no se puede afirmar categóricamente que es positiva, ni cual la dirección del efecto.

El tercer bloque de análisis se centra en el efecto de la apertura comercial en la desigualdad de la renta, a través del incremento en la desigualdad salarial entre trabajadores cualificados y no cualificados. Entre dichos estudios podríamos destacar los de Krugman (1995), Lawrence (1996), Cline (1997), Borjas, Freeman y Katz (1997). Estos trabajos se basan en la aplicaciōn del conocido teorema de Stolper-Samulson. Como los países industrializados han empezado a competir con paîses menos avanzados con salarios más reducidos 
abundantes en trabajadores no cualificados, el resultado es un aumento del trabajo cualificado y una reducción en el no cualificado en los países industrializados con el consecuente efecto en los salarios.

El propio Krugman (2008), critica esos resultados, ya que su obtención se ha realizado utilizando datos del corto plazo, cuando el modelo teórico que lo sustenta es un modelo de largo plazo. Además, el autor revisa el efecto del comercio sobre los salarios en Estados Unidos, encontrando una escasa cuando no insignificante relaciōn entre el comercio de mercancías con China, principalmente, y la desigualdad salarial. La explicación de esta última se traslada al progreso técnico.

Se ha seguido trabajando en esta línea, con más refinamientos empíricos, y centrāndose más en aspectos microeconómicos que macroeconómicos. Un resumen de las últimas aportaciones, así como una revisión empírica detallada de los modelos se puede ver en Helpman (2016). La conclusión es que la desigualdad no se ha generado por la globalización, sino por el cambio técnico, en el que dicha globalización ha tenido un papel, pero pequeño.

Desde la perspectiva poskeynesiana las cosas parecen más claras en cuanto a los efectos de la globalización sobre el crecimiento y el desempleo. La globalización a partir de las décadas de los 80 y 90 del siglo pasado ha producido que la mayoría de los países industrializados hayan abandonado las políticas de expansiōn de la demanda interna para hacer frente al aumento del desempleo, a favor de las políticas basadas en la expansión de exportaciones netas. Esas políticas han conducido a una reducción generalizada de los salarios, de forma que la renta distribuida como pago al factor trabajo ha disminuido. La mayor propensión al consumo de los salarios que de los beneficios, reduce la demanda de consumo, que disminuye el crecimiento de la demanda agregada, salvo que esa bajada se compense con una subida de las exportaciones netas y la inversiōn 7 . Si se cumple la restricción externa (la ley de Thriwal) sobre las exportaciones netas a largo plazo, y las políticas fiscales restrictivas de los 80 y 90, la implicación de los economistas poskeynesianos es que la globalización a través del efecto de los salarios ha conducido a menores tasas de crecimiento y un mayor desempleo. Este aumento del desempleo se debe a que la demanda de trabajo no crece lo suficiente para absorber el crecimiento de la población activa, por la diferencia entre la tasa de crecimiento de la población y la garantizada por la demanda. El desempleo es endógeno y determinado por la demanda.

A partir de las conclusiones de los numerosos estudios realizados sobre el tema, en este trabajo trataremos de contrastar algunos de los resultados que se han dado en la literatura, que resumimos a continuación, sobre las diferentes variables:

7 Es la definiciōn, a travês del modelo de Badhuri-Marglin (1990), de la división de los paîses por el efecto de la disminución de los salarios sobre la demanda agregada entre del lado de los salarios (o de los beneficios) si la disminuciōn salarial disminuye (aumenta) la demanda agregada. 
1. Tasa de apertura. La gran mayoría de los trabajos sobre el tema parten de la hipótesis de que la globalización se ha intensificado a partir de finales de los 80 del siglo pasado. Eso se traduce en un incremento de los volúmenes de comercio internacional, tanto por la incorporación de un mayor número de países, como por la eliminación de la mayoría de las barreras al comercio. Una variable que mide dicha situación es la tasa de apertura de la economía, que si la hipótesis es correcta, habría aumentado su tasa de crecimiento en esas fechas.

2. La tasa de crecimiento del PIB y de la productividad. En el caso de esta variable las conclusiones son diferentes según se planteen desde la economía ortodoxa o desde los poskeynesianos. Para los primeros, el aumento de la globalizaciōn habría aumentado la productividad, y como consecuencia la tasa de crecimiento. Para los segundos, debido a su efecto sobre los salarios, habría sucedido lo contrario. En ambos casos las tasas de crecimiento de la producción y la productividad habrían sufrido un cambio en torno a esas fechas.

3. Tasa de desempleo. En este caso, tambiēn son diferentes las conclusiones obtenidas por el modelo ortodoxo o por los keynesianos. Para los primeros, aunque pudiera haber efectos en el empleo a corto plazo, a largo plazo la economía volverá al empleo de equilibrio y la tasa de desempleo a la tasa natural de paro, puesto que esta no depende de estos factores. Para los keynesianos, no existe la tasa natural de paro, y los efectos a corto plazo se trasladan al largo plazo puesto que la tasa de desempleo depende de la demanda agregada. Un menor crecimiento de la demanda aumenta el desempleo.

En este trabajo, utilizando las técnicas de estacionaridad de las series de tiempo vamos a tratar de contrastar empíricamente si esas predicciones son correctas, como veremos en el siguiente apartado. Para ello utilizaremos datos sobre esas variables para un grupo numeroso de países miembros de la Uniōn Europea. La elecciōn de estos países se realiza por el hecho de que se dan en ellos todos los factores propios de la globalizaciōn.

Por una parte, todos son países dentro del grupo de países industrializados de la OCDE. Además, son miembros de la Uniōn Europea que es un espacio caracterizado por la libertad de comercio de mercancías y servicios, de capitales y de personas. Por último, a partir de mediados de los 90 se incorporaron a la Uniōn Europea los países del este europeo que provenían del antiguo bloque socialista, cuyos salarios eran muy inferiores a los paises de europa occidental, y que entraron en la zona de libre comercio con el resto ${ }^{8}$. Son trece países cuya forma de integraciōn en la Uniōn Europea es diferente, por lo que su participación también. Entre ellos, diez pertenecen a la zona euro, y tres conservan la moneda propia. Hemos prescindido de algunos países porque su tamaño es muy pequeño. 
No hemos abordado el efecto de la globalización sobre la distribución por cuestiones de extensiōn, pero quedaría como un tema futuro de investigaciōn.

\section{Metodolocía}

Para analizar empíricamente la relaciōn entre la apertura comercial, el crecimiento y el empleo (desempleo) vamos a emplear una metodología indirecta. Como la comprobación directa ya se ha visto que desde el punto de vista macroeconómico es muy complicada cuando no imposible, optamos por esta otra vía.

La metodología estadística que utilizaremos es comprobar si las series a analizar son estacionarias o no. La estacionaridad de la serie implica que existe un valor de equilibrio al que la serie tiende a largo plazo. Dicha estacionaridad puede ser en la media o en la tendencia. En el caso que nos ocupa, lo que nos interesa ver es si las series son estacionarias en la media. Es decir, si existe una tasa de crecimiento o de desempleo de equilibrio a largo plazo. Si la variable no es estacionaria (presenta una raíz unitaria), es que dicha variable no tiene un valor de equilibrio a largo plazo.

El procedimiento habitual para comprobar si una serie es estacionaria es responder a la pregunta: ¿es la serie estacionaria o se representa de forma autorregresiva conteniendo una o varias raíces unitarias? Para responder a dicha pregunta existen una serie de test que permiten rechazar o aceptar las hipótesis de estacionaridad de dichas series de tiempo. Los primeros test usados, los de Dickey-Fuller, Phillips-Perron y el Augmented DickeyFuller, tenían poca capacidad predictiva para rechazar la hipótesis nula de no estacionaridad, es decir rechazar que la variable fuese I(1). En particular esto se hacía más importante si la serie presentaba cualquier ruptura estructural (cambio en el valor de la media) incluso cuando la serie era estacionaria antes y después de dicho cambio.

Es por ello que los investigadores desarrollaron test más potentes, como el DF-GLS de Elliot, Rothenberg y Stock (1996). El test de Dickey y Fuller es una regresiōn mínimo-cuadrática (OLS), el Dickey y Fuller aumentado (ADF) es una regresión OLS con retardos, y el DFGLS es el ADF con mínimos cuadrados generalizados (GLS). Dicha regresión, supuesto que no hay tendencia y, por tanto, es estacionaria alrededor de la media, consiste en:

$$
\Delta y_{t}^{d}=\alpha+\rho y_{t-1}^{d}+\sum_{i=1}^{m} \delta_{i} \Delta y_{t-1}^{d}+\varepsilon_{t}
$$

Donde $m$ es el número de retardos. La variable $y_{t-1}^{d}$ es la variable después de quitarle la tendencia. Los valores críticos para rechazar la hipótesis de I (1), cuando el valor del estadístico t, es mayor de un determinado valor, generalmente proporcionado por los programas econométricos al uso (en nuestro caso Stata versión 15). 
Pero muchas veces los test de raíces unitarias no son capaces de rechazar la hipótesis nula en el caso de que exista una ruptura, bien en la tendencia, en la media o en ambas, siendo la serie estacionaria antes y después de la perturbaciōn que provocó esa ruptura. Perron (1989) construyó un test que, permitiendo un cambio exógeno en la media, permitía rechazar la hipótesis de raíz unitaria en las series de largo plazo del crecimiento del PIB, en las que, Nelson y Plosser (1982), no pudieron hacerlo. Más adelante, los trabajos de Banerjee, Lumsdaine, and Stock (1992) y Zivot y Andrews (1992), endogeinizan el periodo de la ruptura. Perron y Voselang (1992) construyen un test que permite dos formas de introducir la ruptura en la media, el modelo aditivo (additive outlier AO) que captura un cambio repentino, y el modelo innovacional (innovative outlier, IO) que recogería un cambio gradual.

El modelo AO considera que la dinámica de la variable viene dada por:

$$
y_{t}=\delta D T_{b t}+y_{t-1}+w_{t}
$$

Con $D T_{b t}=1$ para $t=t_{b}+1$, 0 en otro caso, bajo la hipótesis nula de una raíz unitaria. En el caso de la hipótesis contraria:

$$
y_{t}=c+\delta D U_{t}+v_{t}, \mathrm{t}=2, \ldots, \mathrm{T}
$$

Donde $D U_{t}=1$ para $t>t_{b}, 0$ en el resto.

La especificaciōn general hace recaer la hipótesis nula (2) en el caso de que la distribución de $v_{t}$ pueda descomponerse entre una raíz unitaria y un proceso autorregresivo (ARMA). Se trata, por tanto, de estimar la ecuación:

$$
y_{t}=\mu+\delta D U_{t}+\tilde{y}_{t}
$$

Los residuos $\left(\tilde{y}_{t}\right)$ se regresan sobre sus valores retardados y un conjunto de variables dummy, esto último para asegurar que la distribuciōn del estadístico puede ser manejada:

$$
\tilde{y}_{t}=\sum_{i=0}^{k} \omega_{i} D T_{b t-i}+\alpha \tilde{y}_{t-i}+\sum_{i=1}^{k} \theta_{i} \Delta \tilde{y}_{t-i}+e_{t}, \mathrm{t}=\mathrm{k}+2, \ldots, \mathrm{T}
$$

Perron y Voselang proporcionan los valores críticos del estadístico t de $\alpha$ para rechazar la hipótesis nula de I(1).

El modelo IO presenta la ruptura como un efecto dinámico de $D T_{b}$ tiene una representación autorregresiva finita (AR):

$$
y_{t}=\mu+\delta D U_{t}+v D T_{b t}+\alpha y_{t-1}+\sum_{i=1}^{k} \theta_{i} \Delta y_{t-i}+e_{t}, \mathrm{t}=\mathrm{k}+2, \ldots, \mathrm{T}
$$


Tanto en el modelo AO como en el modelo IO el valor del periodo de ruptura $\mathrm{T}_{\mathrm{b}}$ como el número de retardos $\mathrm{k}$ son desconocidos. La forma de hacerlo es ir estimando la ruptura en cada período y luego elegir el valor óptimo siguiendo una de las diversas reglas para identificar la ruptura. El orden de retardos se realiza mediante el método propuesto por Perron (1990).

Esta metodología ha sido extendida para una doble ruptura de la media por Clemente, Montañés y Reyes (1998). La búsqueda de la doble ruptura (puntos $\mathrm{T}_{\mathrm{b} 1} \mathrm{y}_{\mathrm{b} 2} \mathrm{~T}_{\mathrm{b}}$ ) puede realizarse tanto en el modelo $\mathrm{AO}$ como en el IO. El modelo AO requiere la estimación de:

$$
y_{t}=\mu+\delta_{1} D U_{1 t}+\delta_{2} D U_{2 t}+\tilde{y}_{t}
$$

Y la búsqueda secuencial del valor mínimo del estadístico t para la hipótesis $\alpha=1$ en el modelo:

$$
\tilde{y}_{t}=\sum_{i=0}^{K} \omega_{i} D T_{b 1, t-i}+\sum_{i=0}^{K} \omega_{i} D T_{b 2, t-i}+\alpha \tilde{y}_{t-1}+\sum_{i=1}^{k} \theta_{i} \Delta \tilde{y}_{t-i}+e_{t}, \mathrm{t}=\mathrm{k}+2, \ldots, \mathrm{T}
$$

Para el modelo IO sigue el proceso similar a la ecuaciōn (6) en el esquema de la (8).

Esta metodología la aplicamos en nuestro caso a las variables antes indicadas, tasa de apertura, tasas de crecimiento del PIB y de la productividad, y la tasa de desempleo. En todos los casos, excepto en la de desempleo hemos obtenido la tasa de crecimiento, en términos reales, y hemos realizado el test de estacionaridad de cada una. En el supuesto de que las variables sean estacionarias, esto implicaría que existe una tasa constante, que es la tasa de equilibrio de largo plazo. Si alguna perturbación ha hecho que dicha tasa presente un cambio en el equilibrio, los test nos permitirán conocer la fecha de la ruptura, lo que nos permitirá contrastar si coincide, tanto en el período como en el signo con lo predicho por la literatura.

Para obtener los datos de las variables hemos utilizado la base de datos AMECO de la Comisión Europea. Esta base de datos presenta series de cada una de las variables: Exportaciones, Importaciones, PIB, Productividad Media, en términos constantes de 2010, y la tasa de desempleo, desde 1960 hasta 2017 lo que permite un análisis de largo plazo.

Los resultados obtenidos para cada una de las variables, debido a los continuos test de estacionaridad que han de realizarse, se presentan en cuadros resumen, donde se indica si la variable es estacionaria o no. En el caso de que no lo sea mediante el test de Dickey y Fuller generalizado, se realiza los test de una ruptura de la media, tanto del modelo AO como IO. Presentamos el resultado como la fecha de ruptura. Si aún sigue sin ser estacionaria, realizamos el test con dos rupturas, igualmente en ambos modelos. En el caso de que sigan sin ser estacionarias, asumimos que no existe una tasa de equilibrio. Los diferentes test, que no se presentan en este trabajo por la extensiōn que conllevarian, los autores los tienen a disposición de las personas que lo soliciten. Los resultados obtenidos para cada variable se presentan en el siguiente apartado. 


\section{Resultados EMPÍRICOS}

Antes de comentar los resultados obtenidos para cada una de las variables, describiremos brevemente la evolución de cada una de ellas. En el anexo 1 se presentan los gráficos de cada variable. Debido a que estudiamos 14 países, presentamos cada variable en dos gráficos con 7 países, por orden alfabético, en cada uno. Las series las hemos obtenido de la base de datos de la AMECO (Comisión Europea), y nos proporciona series con base en los precios de 2010 desde 1960 hasta 2017. Los datos presentan una extensión de 57 años que nos aportan series largas de tiempo para analizar los efectos a largo plazo.

Los gráficos 1A y 1B representan la tasa de apertura, calculada como la ratio entre la suma de exportaciones más importaciones en términos reales divido por el PIB real. Como puede observarse en dichos gráficos, la tasa de apertura ha crecido de forma continuada desde el año 1960. Los países más pequeños presentan tasas de apertura mayores, de hecho, Holanda, Bélgica, Dinamarca, Austria e Irlanda presentan tasas, en 2017, por encima del 100\%, y en el caso de Irlanda por encima del 200\%. Los países grandes están todos en torno al 65\%, excepto Alemania que tiene el 92\%. Portugal y Grecia son países pequeños con una tasa de apertura menor del $100 \%$.

El valor de las tasas de apertura está condicionado claramente por el tamaño del país, por lo que tomada por sí misma no parece un indicador fiable. Es por ello, que el análisis se centra, no en el valor, sino en la tendencia, es decir, su tasa de crecimiento.

Los gráficos 2A y 2B, y los 3A y 3B muestran las tasas de crecimiento de la productividad media y del PIB real respectivamente. Se puede apreciar que desde 1960 presentan una tendencia decreciente, que luego trataremos de ver si es un efecto de corto o de largo plazo. Existe una reducción bastante grande desde 1960 respecto de finales de los 70, y luego no parece clara su evolución.

Por último, en los gráficos 4A y 4B se refieren a las tasas de desempleo. La dirección de dichas tasas se relaciona claramente con la evolución de la tasa de crecimiento, ya que se observa un claro aumento del desempleo en la mayoría de los países. Igual que en el caso anterior, parece que el salto se observa a finales de los años 70, principios de los 80. En esta ocasión no parece observarse una tendencia clara, pero un análisis en profundidad de los datos nos permitirá extraer algunas conclusiones.

A primera vista podríamos decir que desde 1960 ha crecido la tasa de apertura de forma considerable, al mismo tiempo que en las economías se producía una caída de las tasas de crecimiento de la productividad y de la producción, y a su vez se originaba un aumento de las tasas de desempleo. En este caso, se puede afirmar que los datos van en línea con lo mantenido por los poskeynesianos. Los ortodoxos interpretarían, en cambio, que las tasas de crecimiento han disminuido y las tasas de paro han aumentado debido a las políticas de demanda que se habían mantenido hasta mediados de los 80, y, que a partir de aquí, los cambios en la globalización, junto con las reformas de los mercados de trabajo han hecho que dichas tasas no hayan empeorado más. 
La hipótesis en la cual pivota lo manifestado anteriormente, es que ha existido un cambio en la tasa de crecimiento de la globalización, que ha conllevado una variación en las tasas de crecimiento y de desempleo de largo plazo $^{9}$, que se comprobará si es correcta en los apartados siguientes.

\section{1. LA TASA DE APERTURA}

La primera de las hipótesis que vamos a tratar de comprobar es aquella por la cual se plantea que la globalizaciōn se ha intensificado desde finales de los años 80 y principios de los 90 respecto a épocas anteriores. Para ello, se supone que esa intensificación se corresponde con un aumento en la tasa de crecimiento de las variables que miden la globalización, y en concreto, un aumento del volumen de comercio. Dicho volumen se mide como la suma de las exportaciones y las importaciones de las diferentes economías, de forma relativa respecto del PIB, todo en términos reales, lo que se denomina la tasa de apertura.

Dicha tasa de apertura ha estado creciendo de forma continuada desde el final de la Segunda Guerra Mundial, como se puede ver en los gráficos $1 \mathrm{~A}$ y $1 \mathrm{~B}$, por lo que la intensificación la podemos definir como un aumento de dicha tasa de crecimiento. En términos estadísticos que nos ocupa un cambio en la media.

En el cuadro 1 se presentan los resultados de aplicar la metodología sobre la tasa de crecimiento de la apertura comercial. Primero, se analiza si la serie es estacionaria. En este caso se observa que para 7 de los 14 paîses la tasa de apertura muestra un crecimiento constante alrededor de un valor de equilibrio, y que no ha habido cambios significativos en dicha tasa. Ese primer grupo de países son Alemania, Bélgica, Finlandia, Francia, Holanda, Irlanda e Italia, que presentan tasas de crecimiento en torno al 2,5\% anual, excepto Irlanda cuya apertura comercial ha crecido a un ritmo muy elevado desde 1960, al 3,7\%.

Cuando la serie no era estacionaria, se analizaba si existía una ruptura en la media, tanto con el modelo $\mathrm{AO}$ como con el modelo IO, comprobándose, en cada caso que la serie era estacionaria antes y después de la ruptura. En el cuadro aparece el resultado con el año que el test selecciona como de ruptura. En este caso, otros 6 países muestran que la serie es estacionaria con una ruptura. El período de ruptura es diferente en cada país, aunque en 4 de ellos, Austria, Dinamarca, España y Reino Unido, sus fechas están en torno a mediados de los 2000, cuando los países del antiguo bloque soviético entraron a formar parte de la Unión Europea. Pero al contrario de lo esperado, el crecimiento de la tasa de apertura se ha reducido en más de la mitad y no ha aumentado, como suponen la mayoría de los trabajos.

9 La contrastación de diversas teorías sobre los modelos de crecimiento y la existencia de la tasa de paro de equilibrio se puede encontrar en Papell et al. (2000) y Pappell y Prodan (2011). 
Suecia es el único país que presenta dos rupturas de la media, pero teniendo en cuenta la profunda crisis que sufrió en los años 90, donde se encuentran las rupturas, y que ambas se localizan en un entorno de 6 años, quizás podríamos descartarlas como un efecto estadístico, frente a la hipótesis de que presente una serie estacionaria para todo el período.

CuAdro 1. TASA DE APERTURA

\begin{tabular}{|c|c|c|c|c|}
\hline PAIS & ESTACIONARIA & RUPTURAS & AÑOS & TASA DE EQUILIBRIO \\
\hline ALEMANIA & $\mathrm{SI}$ & NO & & $2,78 \%$ \\
\hline \multirow[t]{2}{*}{ AUSTRIA } & $\mathrm{SI}$ & 1 & 2007 & $2,95 \%$ \\
\hline & & & & $1,22 \%$ \\
\hline BELGICA & $\mathrm{SI}$ & NO & & $2,46 \%$ \\
\hline \multirow[t]{2}{*}{ DINAMARCA } & $\mathrm{SI}$ & 1 & 2006 & $2,42 \%$ \\
\hline & & & & $1,47 \%$ \\
\hline \multirow[t]{2}{*}{ ESPAÑA } & $\mathrm{SI}$ & 1 & 2007 & $3,95 \%$ \\
\hline & & & & $0,82 \%$ \\
\hline FINLANDIA & $\mathrm{SI}$ & NO & & $2,08 \%$ \\
\hline FRANCIA & $\mathrm{SI}$ & NO & & $2,89 \%$ \\
\hline \multirow[t]{2}{*}{ GRECIA } & $\mathrm{SI}$ & 1 & 1999 & $3,59 \%$ \\
\hline & & & & $2,09 \%$ \\
\hline HOLANDA & $\mathrm{SI}$ & NO & & $2,72 \%$ \\
\hline IRLANDA & $\mathrm{SI}$ & NO & & $3,70 \%$ \\
\hline ITALIA & $\mathrm{SI}$ & NO & & $2,76 \%$ \\
\hline \multirow[t]{2}{*}{ PORTUGAL } & $\mathrm{SI}$ & 1 & 1974 & $4,02 \%$ \\
\hline & & & & $3,76 \%$ \\
\hline \multirow[t]{2}{*}{ REINO UNIDO } & $\mathrm{SI}$ & 1 & 2004 & $2,05 \%$ \\
\hline & & & & $1,37 \%$ \\
\hline \multirow[t]{3}{*}{ SUECIA } & $\mathrm{SI}$ & 2 & 1992 & $1,74 \%$ \\
\hline & & & 1999 & $2,12 \%$ \\
\hline & & & & $1,48 \%$ \\
\hline
\end{tabular}

Podemos concluir, que para un grupo mayoritario de 7 países, la tasa de apertura no ha sufrido cambios desde 1960, creciendo de modo constante todo este período. Para 4 de ellos, se produce un cambio, pero ese cambio supone una reducción del crecimiento de dicha apertura. Por último, Suecia presenta su propia estructura. No podemos afirmar, por tanto, que la entrada de nuevos países en el comercio mundial en los años 90 del siglo pasado haya sido algo relevante en el proceso de globalización de los países industrializados de la Unión Europea. 
4.2. TASAS DE CRECIMIENTO DE LA PRODUCCIÓN Y DE LA PRODUCTIVIDAD MEDIA

En los cuadros 2 y 3 se presentan los resultados sobre las tasas de crecimiento de la producción y de la productividad media, ambas medidas en términos reales. Igual que en el análisis anterior, el procedimiento seguido es primero realizar el test de raíz unitaria de la variable. En el caso de que no podamos rechazar la hipótesis nula, lo realizamos con una ruptura de la media, y si aun así podemos rechazar la ruptura lo hacemos con dos. En el cuadro aparece el resultado de la estacionaridad de la variable, de los años de ruptura, y de las tasas de equilibrio en los diferentes períodos.

CUADRO 2. PRODUCTIVIDAD MEDIA

\begin{tabular}{|c|c|c|c|c|}
\hline PAIS & ESTACIONARIA & RUPTURAS & AÑOS & TASA DE EQUILIBRIO \\
\hline ALEMANIA & $\mathrm{SI}$ & NO & & $1,68 \%$ \\
\hline \multirow[t]{3}{*}{ AUSTRIA } & $\mathrm{SI}$ & 2 & 1972 & $5,01 \%$ \\
\hline & & & 2006 & $2,27 \%$ \\
\hline & & & & $0,14 \%$ \\
\hline \multirow[t]{3}{*}{ BELGICA } & $\mathrm{SI}$ & 2 & 1973 & $4,25 \%$ \\
\hline & & & 2006 & $2,11 \%$ \\
\hline & & & & $0,34 \%$ \\
\hline DINAMARCA & $\mathrm{SI}$ & NO & & $1,80 \%$ \\
\hline \multirow[t]{3}{*}{ ESPAÑA } & $\mathrm{SI}$ & 2 & 1975 & $6,39 \%$ \\
\hline & & & 1983 & $2,72 \%$ \\
\hline & & & & $1,15 \%$ \\
\hline \multirow[t]{2}{*}{ FINLANDIA } & $\mathrm{SI}$ & 1 & 1998 & $3,49 \%$ \\
\hline & & & & $0,94 \%$ \\
\hline \multirow[t]{3}{*}{ FRANCIA } & $\mathrm{SI}$ & 2 & 1973 & $5,12 \%$ \\
\hline & & & 1998 & $2,28 \%$ \\
\hline & & & & $0,74 \%$ \\
\hline \multirow[t]{2}{*}{ GRECIA } & $\mathrm{SI}$ & 1 & 1972 & $9,21 \%$ \\
\hline & & & & $2,65 \%$ \\
\hline HOLANDA & $\mathrm{SI}$ & NO & & $1,87 \%$ \\
\hline IRLANDA & $\mathrm{SI}$ & NO & & $3,62 \%$ \\
\hline \multirow[t]{3}{*}{ ITALIA } & $\mathrm{SI}$ & 2 & 1973 & $5,68 \%$ \\
\hline & & & 1999 & $2,20 \%$ \\
\hline & & & & $0,14 \%$ \\
\hline \multirow[t]{2}{*}{ PORTUGAL } & $\mathrm{SI}$ & 1 & 1992 & $4,52 \%$ \\
\hline & & & & $1,15 \%$ \\
\hline \multirow[t]{2}{*}{ REINO UNIDO } & $\mathrm{SI}$ & 1 & 2005 & $2,22 \%$ \\
\hline & & & & $0,4 \%$ \\
\hline SUECIA & $\mathrm{SI}$ & NO & & $2,06 \%$ \\
\hline
\end{tabular}


CUADRo 3. CRECIMIENTO DEL PIB

\begin{tabular}{|c|c|c|c|c|}
\hline PAIS & ESTACIONARIA & RUPTURAS & AÑOS & TASA DE EQUILIBRIO \\
\hline ALEMANIA & $\mathrm{SI}$ & $\mathrm{NO}$ & & $2,64 \%$ \\
\hline \multirow[t]{3}{*}{ AUSTRIA } & $\mathrm{SI}$ & 2 & 1973 & $4,87 \%$ \\
\hline & & & 2007 & $2,76 \%$ \\
\hline & & & & $0,91 \%$ \\
\hline \multirow[t]{2}{*}{ BELGICA } & $\mathrm{SI}$ & 1 & 1973 & $4,83 \%$ \\
\hline & & & & $1,95 \%$ \\
\hline \multirow[t]{2}{*}{ DINAMARCA } & $\mathrm{SI}$ & & 1973 & $4,61 \%$ \\
\hline & & & & $1,75 \%$ \\
\hline \multirow[t]{2}{*}{ ESPAÑA } & $\mathrm{SI}$ & 1 & 1972 & $7,12 \%$ \\
\hline & & & & $2,4 \%$ \\
\hline \multirow[t]{2}{*}{ FINLANDIA } & $\mathrm{SI}$ & 1 & 2006 & $3,48 \%$ \\
\hline & & & & $0,5 \%$ \\
\hline \multirow[t]{2}{*}{ FRANCIA } & $\mathrm{SI}$ & 1 & 1973 & $5,6 \%$ \\
\hline & & & & $1,95 \%$ \\
\hline \multirow[t]{3}{*}{ GRECIA } & $\mathrm{SI}$ & 2 & 1972 & $8,48 \%$ \\
\hline & & & 2007 & $2,88 \%$ \\
\hline & & & & $-2,79 \%$ \\
\hline HOLANDA & $\mathrm{SI}$ & NO & & $2,79 \%$ \\
\hline IRLANDA & $\mathrm{SI}$ & $\mathrm{NO}$ & & $4,84 \%$ \\
\hline \multirow[t]{2}{*}{ ITALIA } & $\mathrm{SI}$ & 1 & 1979 & $4,78 \%$ \\
\hline & & & & $1,23 \%$ \\
\hline \multirow[t]{3}{*}{ PORTUGAL } & $\mathrm{SI}$ & 2 & 1973 & $6,58 \%$ \\
\hline & & & 2000 & $3,19 \%$ \\
\hline & & & & $0,43 \%$ \\
\hline REINO UNIDO & $\mathrm{SI}$ & $\mathrm{NO}$ & & $2,38 \%$ \\
\hline \multirow[t]{3}{*}{ SUECIA } & $\mathrm{SI}$ & 2 & 1969 & $4,37 \%$ \\
\hline & & & 1992 & $2,61 \%$ \\
\hline & & & & $2,5 \%$ \\
\hline
\end{tabular}

Como puede apreciarse, los resultados vuelven a ser distintos para los diferentes países. Para Alemania, Holanda e Irlanda las series son estacionarias, de forma que tanto la tasa de crecimiento de la producción como la de la productividad se han incrementado de forma constante desde 1960. Los demás países han sufrido una perturbaciōn que ha afectado a sus tasas de crecimiento de largo plazo. Muchos de ellos, 5, incluso han sufrido 2 rupturas en la media. Todos han tenido una primera perturbaciōn entre los años 1973 y 1983 que ha afectado a ambas tasas, correspondiendo con el shock del petróleo de esos años ${ }^{10}$, que como puede comprobarse en 8 de los países ha tenido un efecto de largo plazo. Suecia, igual que en el caso anterior, se distingue del resto, tanto por la perturbación, como por el período.

10 En esto es coincidente con otros muchos autores que han encontrado que el shock del petróleo no había tenido efectos de corto plazo sino también de largo plazo. 
En los casos en que la tasa de crecimiento de la productividad no haya cambiado y sí la de producción, podríamos afirmar que ha ocurrido una perturbaciōn de la demanda que provoca efectos de largo plazo. Este parece haber sido el caso de Suecia, en 1992, Portugal en el 2000, Grecia en el 2007. Austria, Bélgica y Reino Unido han tenido una segunda perturbaciōn a mediados de los 2000, coincidiendo con la entrada de los países del este de Europa en la UE. También observamos el mismo efecto en la tasa de crecimiento de la apertura, por lo que parece indicar que la adhesión de los antiguos países del bloque soviético ha tenido alguna influencia en dichos paîses. No asī en el resto.

Lo que parece, a raíz de los resultados, es que tanto las perturbaciones de oferta (productividad) como las de demanda provocan cambios en la tasa de crecimiento de la producción a largo plazo ${ }^{11}$. En ausencia de perturbaciones, la economía crece a una tasa constante en el largo plazo.

\subsection{LA TASA DE DESEMPLEO}

En el cuadro 4 presentamos los resultados sobre la tasa de desempleo de los 14 países en estudio. El desempleo es el resultado de lo que ocurre en el mercado de trabajo en su conjunto, tanto por el lado de la demanda de trabajo, que depende de la tasa de crecimiento de la producción, como de los movimientos de la población activa. Esa dependencia provoca que se vea afectada tanto por las perturbaciones de oferta como las de demanda, puesto que ambas afectan al crecimiento $^{12}$. Como consecuencia, la tasa de desempleo es endógena ${ }^{13}$, o bien, está afectada por lo que se conoce como histéresis. En el primer caso, puede haber una tasa de equilibrio, pero que puede cambiarse con políticas de demanda; en el segundo, no existe tasa de equilibrio y la variable no es estacionaria.

Los datos del cuadro 4 nos muestran que en 6 de los 14 países la tasa de paro no es estacionaria ni con dos perturbaciones en la media. En el caso de Alemania, Holanda e Irlanda la tasa de paro no es estacionaria, a pesar de que la tasa de crecimiento sí lo es, indicando el resultado se debe al efecto de otro tipo de variables que influyen en dicha tasa de paro.

En el resto de países, los resultados indicarían que existe una tasa de equilibrio, con una o dos perturbaciones en la media. Suecia y Finlandia presentan un aumento de la tasa de desempleo como consecuencia de la recesión de demanda de mediados de los 90 en ambos países, como consecuencia de la caída del bloque soviético. En términos de tasa de apertura

11 Lo que contradice el modelo neoclásico del crecimiento económico en el que sólo los cambios en la productividad provocarían cambios en la tasa de crecimiento de la producción.

12 En el apartado anterior se observó que la tasa de crecimiento de largo plazo se ha visto afectada por ambos tipos de perturbaciones.

13 Tanto los neoclásicos como los neokeynesianos aceptan la hipótesis de una tasa de desempleo de equilibrio, tasa natural de paro o NAIRU correspondientemente. 
esa caída afectó más al comercio de Suecia que al de Finlandia, que parece más afectada por la caída de empresas como, por ejemplo, Nokia.

La perturbación de los precios del petróleo de finales de los 70 y principios de los 80 también ha tenido como consecuencia un aumento en las tasas de desempleo de varios de los países. Ese ha sido el caso de Bélgica, Dinamarca, España, Italia, Portugal y Reino Unido. El caso de España es muy llamativo, porque pasa del 4,85\% al 16,88\%, pero en el resto, los aumentos son también de una cuantía importante.

CUADRO 4. TASA DE DESEMPLEO

\begin{tabular}{|c|c|c|c|c|}
\hline PAIS & ESTACIONARIA & RUPTURAS & AÑOS & TASA DE EQUILIBRIO \\
\hline ALEMANIA & $\mathrm{NO}$ & & & NO \\
\hline AUSTRIA & NO & & & $\mathrm{NO}$ \\
\hline \multirow[t]{3}{*}{ BELGICA } & $\mathrm{SI}$ & 2 & 1973 & $1,92 \%$ \\
\hline & & & 1979 & $6,54 \%$ \\
\hline & & & & $8,43 \%$ \\
\hline \multirow[t]{2}{*}{ DINAMARCA } & $\mathrm{SI}$ & 1 & 1979 & $2,12 \%$ \\
\hline & & & & $6,22 \%$ \\
\hline \multirow[t]{2}{*}{ ESPAÑA } & $\mathrm{SI}$ & 1 & 1983 & $4,81 \%$ \\
\hline & & & & $16,88 \%$ \\
\hline \multirow[t]{3}{*}{ FINLANDIA } & $\mathrm{SI}$ & 2 & 1989 & $3,39 \%$ \\
\hline & & & 1999 & $6,4 \%$ \\
\hline & & & & $8,38 \%$ \\
\hline FRANCIA & NO & & & $\mathrm{NO}$ \\
\hline GRECIA & NO & & & $\mathrm{NO}$ \\
\hline HOLANDA & NO & & & NO \\
\hline IRLANDA & NO & & & $\mathrm{NO}$ \\
\hline \multirow[t]{2}{*}{ ITALIA } & $\mathrm{SI}$ & 1 & 1983 & $5,65 \%$ \\
\hline & & & & $9,48 \%$ \\
\hline \multirow[t]{3}{*}{ PORTUGAL } & $\mathrm{SI}$ & 2 & 1973 & $2,48 \%$ \\
\hline & & & 2007 & $6,88 \%$ \\
\hline & & & & $12,37 \%$ \\
\hline \multirow[t]{3}{*}{ REINO UNIDO } & $\mathrm{SI}$ & 2 & 1977 & $2,12 \%$ \\
\hline & & & 1998 & $5,71 \%$ \\
\hline & & & & $5,88 \%$ \\
\hline \multirow[t]{2}{*}{ SUECIA } & $\mathrm{SI}$ & 1 & 1994 & $2,58 \%$ \\
\hline & & & & $7,44 \%$ \\
\hline
\end{tabular}

En resumen, no se puede establecer que el incremento de la tasa de paro se deba al efecto del aumento de la globalización puesto que los cambios en la tasa de paro se dan en fechas diferentes a la entrada de los países menos industrializados en el comercio de mercancías. 


\section{CONCLUSIONES}

En este trabajo hemos tratado de comprobar empíricamente la relación entre globalizaciōn, crecimiento y desempleo que ha sido y sigue siendo objeto de polémica tanto a nivel académico, como, sobre todo, en la opinión pública. Para ello hemos realizado una breve revisión de lo que la literatura académica ha concluido hasta la fecha, tanto desde la posición ortodoxa como la de los poskeynesianos. El contraste lo hemos realizado de forma indirecta, sobre los modelos que cada uno de ellos proponen, y la causalidad de las variables implicadas. Si la globalizaciōn ha supuesto cambios en las tasas de crecimiento y en el desempleo éstos tienen que verse reflejados en las series de largo plazo de dichas variables. Pasamos a enumerar las principales conclusiones que pueden extraerse del mismo:

1) Las tasas de crecimiento de la producción y de la productividad han sufrido perturbaciones, que han cambiado el valor de equilibrio a largo plazo. La existencia de una tasa de equilibrio parece confirmar la hipótesis del modelo neoclásico, pero en contra de sus supuestos las perturbaciones de demanda también pueden afectar a dicha tasa. Parece, pues, que la tasa de crecimiento depende tanto de la oferta como de la demanda, por lo que se necesita un nuevo modelo de crecimiento que se ajuste a los datos.

2) La tasa de crecimiento de la apertura comercial no ha sufrido cambios en la mayoría de los países, y en los que sí los han sufrido ha sido de una reducción. Dicha caída ha coincidido con un cambio en una menor la tasa de crecimiento a largo plazo. Existe una correlación positiva entre ambas, pero sólo cuando disminuye, lo que indicaría que un mayor aumento de la tasa de apertura no parece afectar a la tasa de crecimiento, pero una reducción de dicha tasa sí que afecta negativamente al crecimiento a largo plazo.

3) La tasa de desempleo es endógena y no existe una tasa de equilibrio. Por tanto, la mayoría de las perturbaciones afectan a dicha tasa, especialmente la tasa de crecimiento. Lo que parece claro es que el aumento que experimentan las tasas de desempleo en los países analizados no se debe a la extensión de la globalización en los años 80 y 90 , sino a factores internos en los diferentes países que afectan al mercado de trabajo. 
Anexo 1. Grāficos

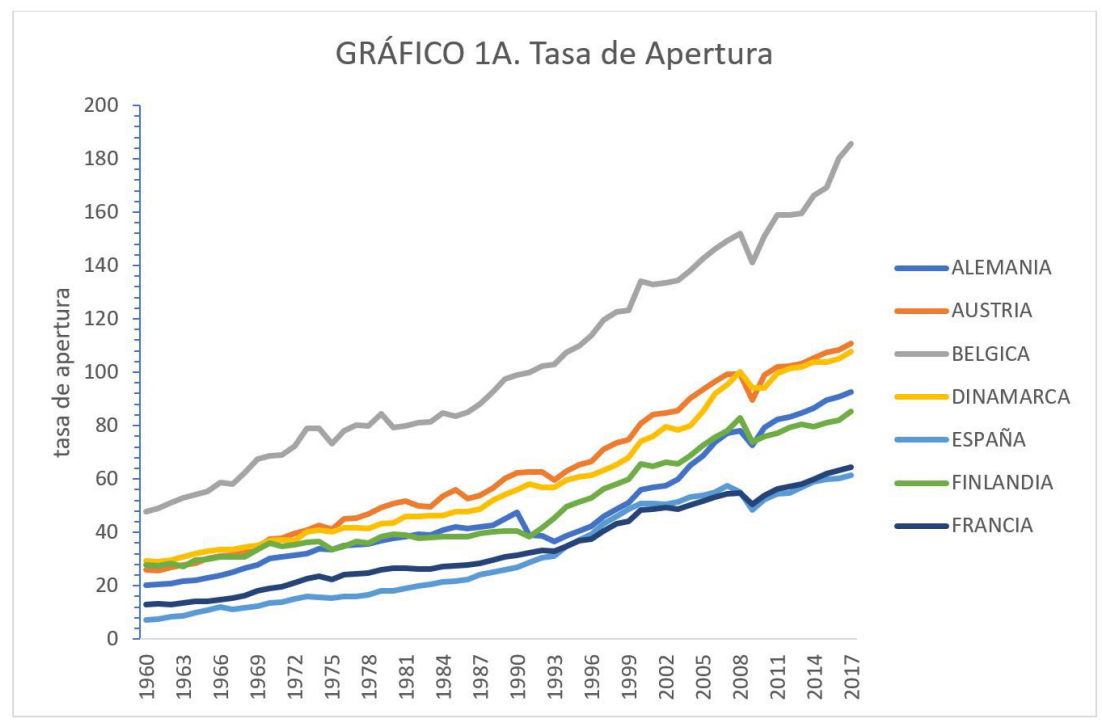

Fuente: Elaboraciōn propia con datos de AMECO

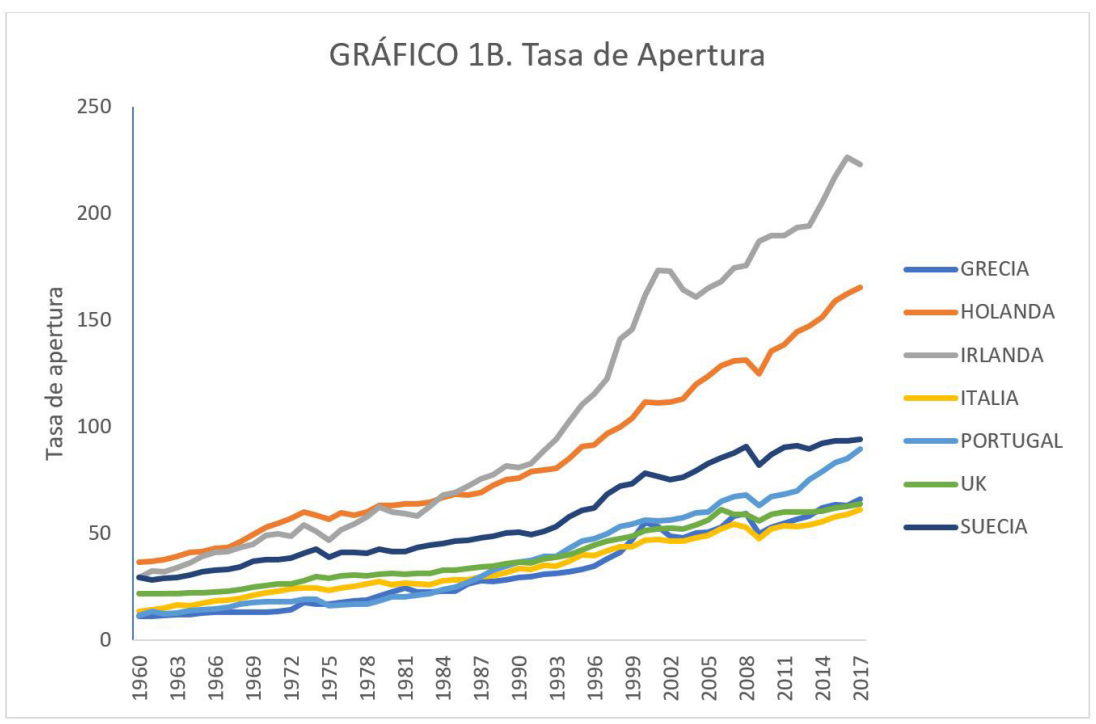

Fuente: Elaboraciōn propia con datos de AMECO 


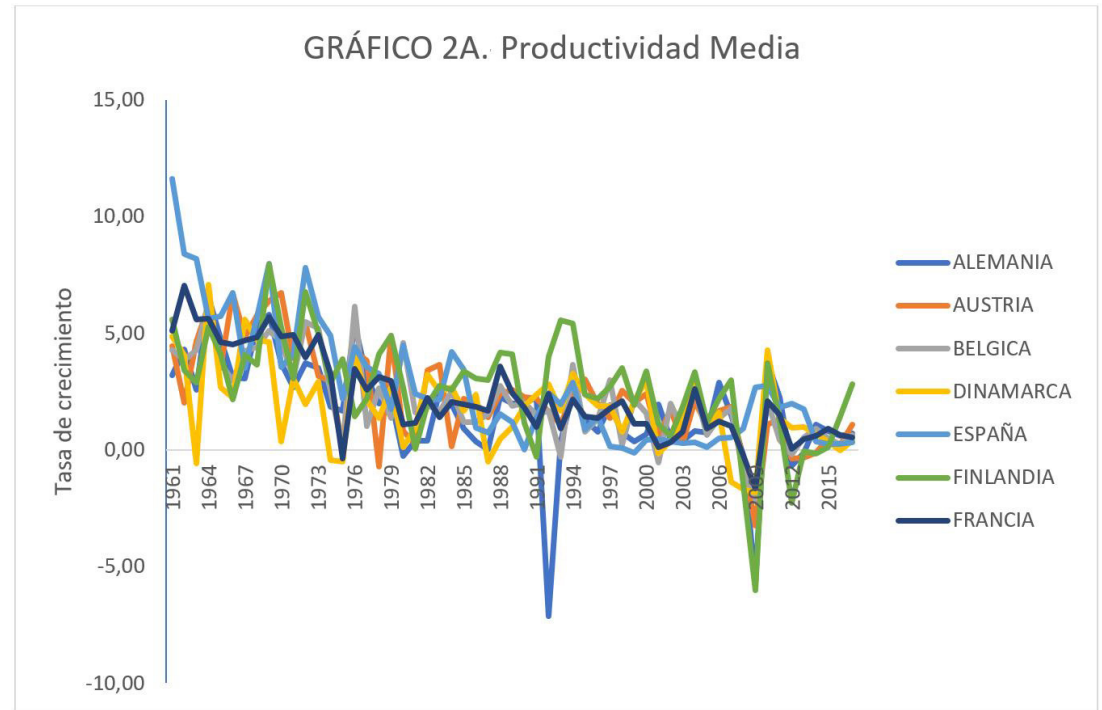

Fuente: Elaboraciōn propia con datos de AMECO

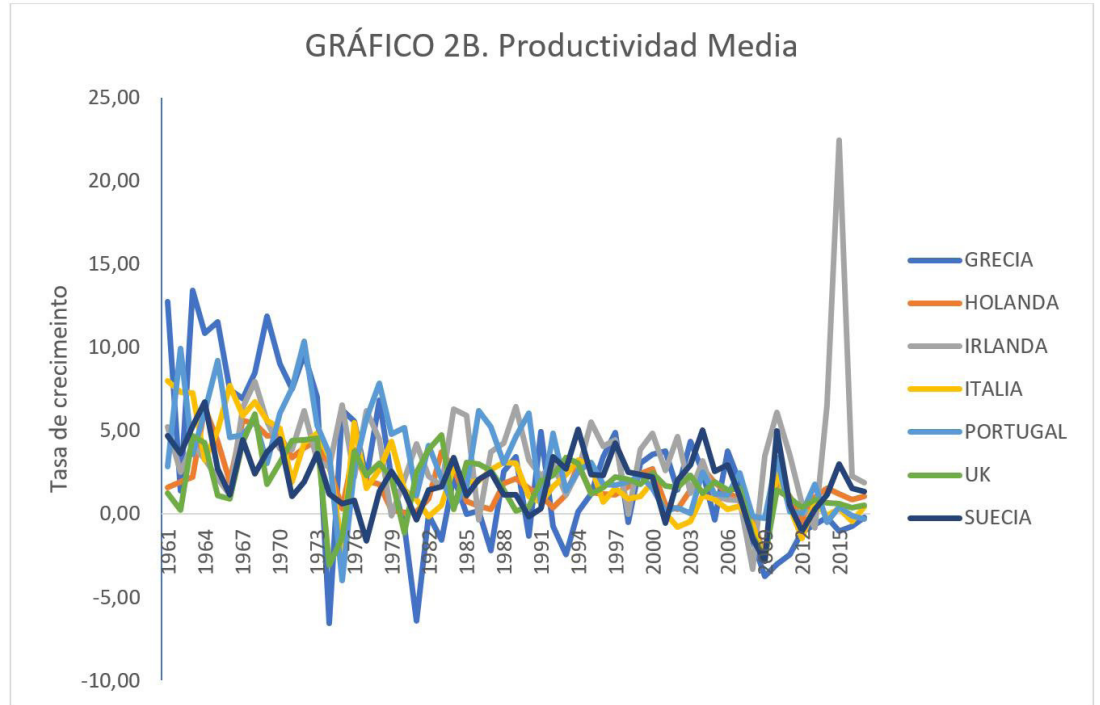

Fuente: Elaboración propia con datos de AMECO 


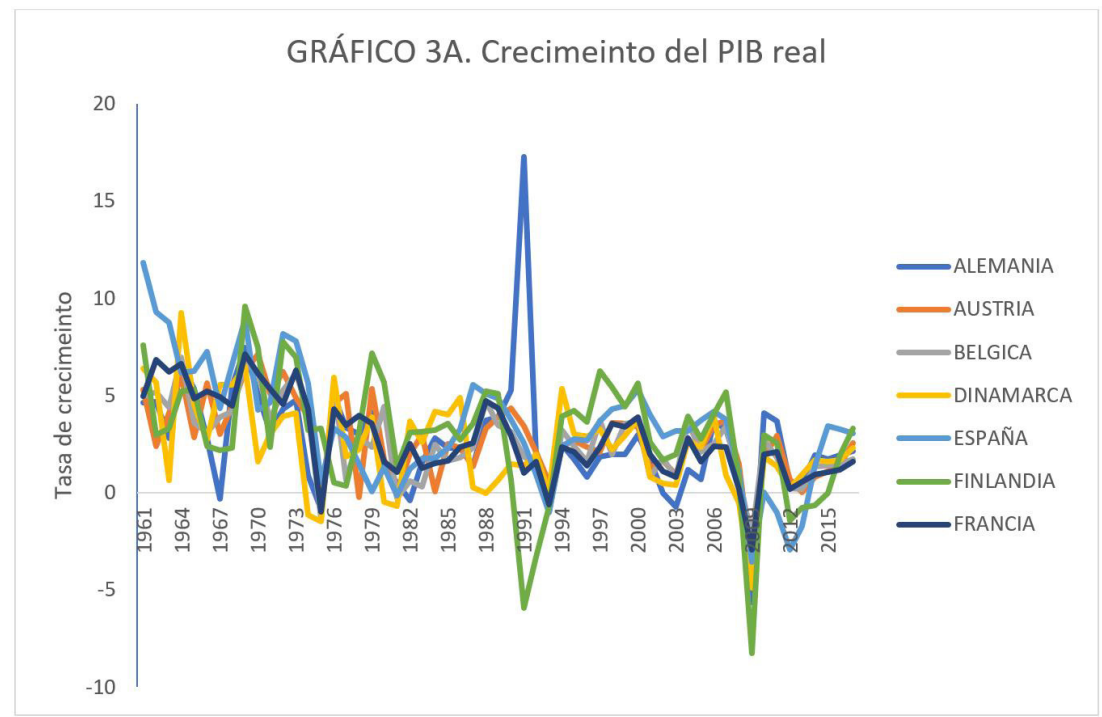

Fuente: Elaboraciōn propia con datos de AMECO

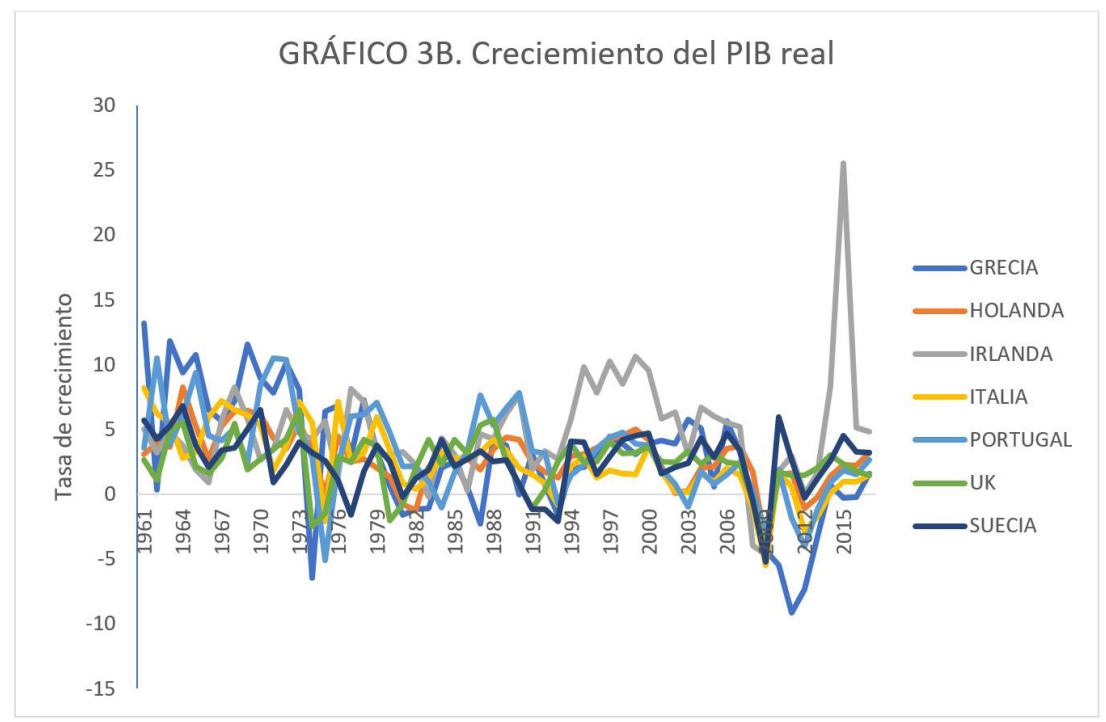

Fuente: Elaboraciōn propia con datos de AMECO 


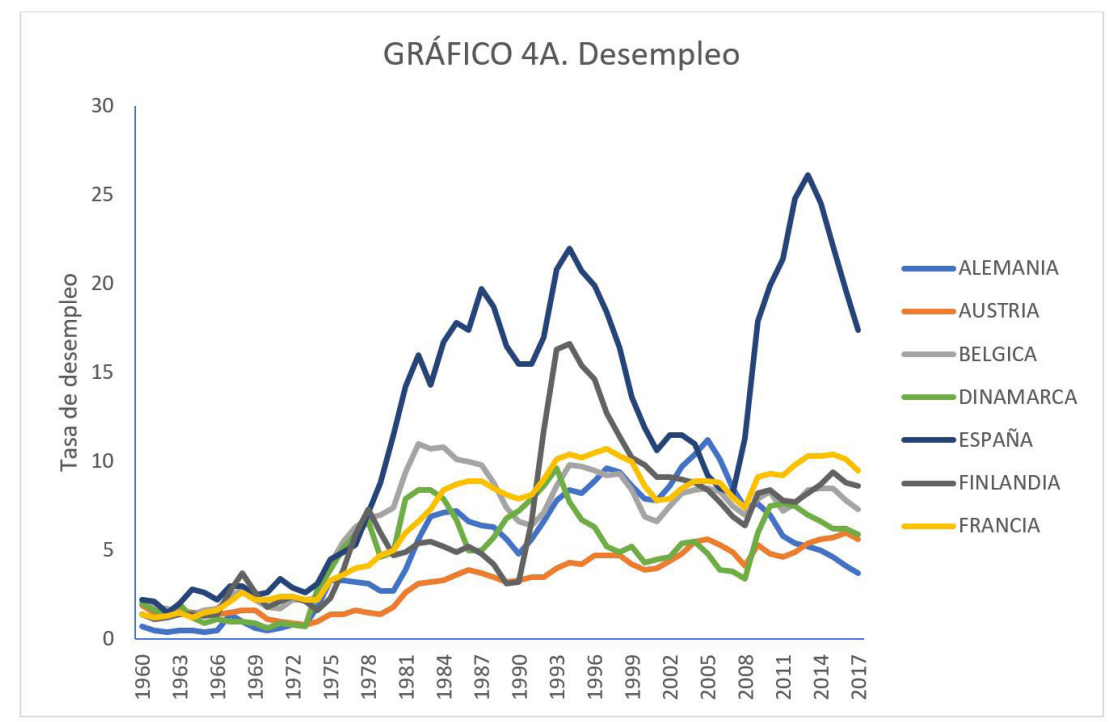

Fuente: Elaboraciōn propia con datos de AMECO

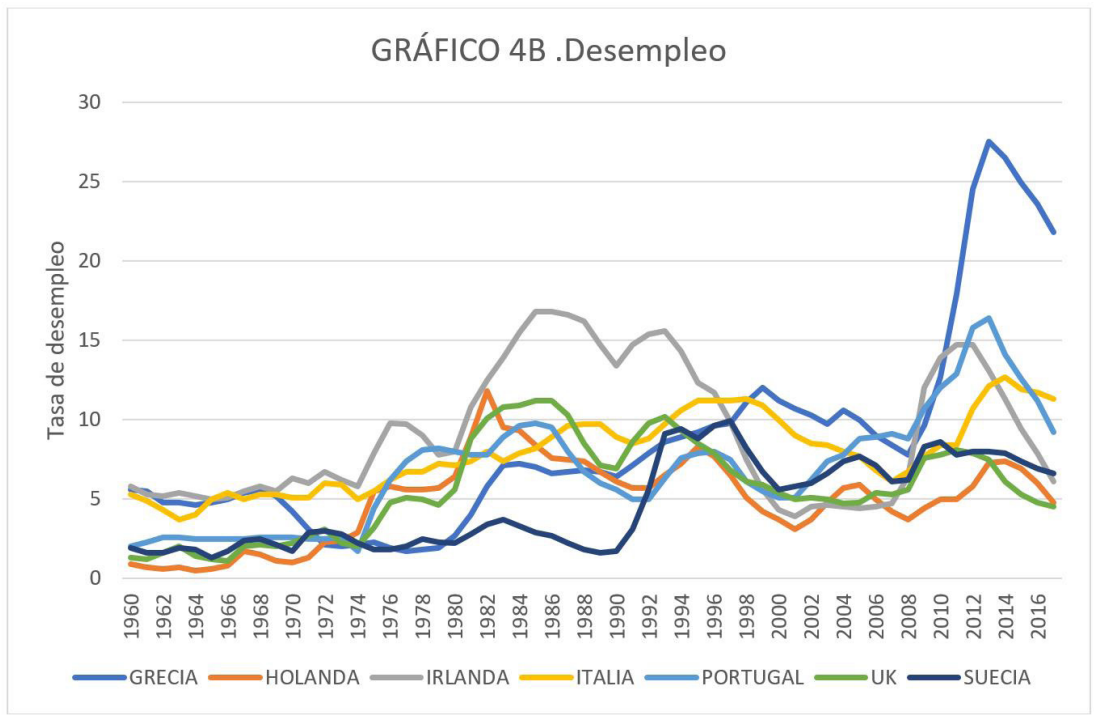

Fuente: Elaboraciōn propia con datos de AMECO 


\section{REFERENCIAS}

Balassa, B. (1985): "Exports, Policy Choices, and Economic Growth in Developing Countries after 1973 Oil Shock". Journal of Development Economics 18(2), 23-35.

Ben-David, D. (1993): “Equalizing Exchange: Trade Liberalization and Income Convergence”. Quaterly Journal of Economics 108(3), 653-679.

Dollar, D. (1992): "Outward-Oriented Developing Economies Really Grow More Rapidly: Evidence from 95 LDCs, 1976-1985". Economic Development and Cultural Change 1992, 523-544.

Bai, J., and P. Perron, (1998): "Estimating and Testing Linear Models with Multiple Structural Changes," Econometrica, 66, 47-78.

Borjas, Richard B. Freeman, and Lawrence F. Katz (1997): "How Much Do Immigration and Trade Affect Labor Market Outcomes?". Brooking Papers on Economic Activity, 1, 1-90.

Bowles, S. y R. Boyer (1995): "Wages, Aggregate Demand, and Employment in an Open Economy: An Empirical Investigation". In Epstein, G. Gintis, H., ed. Macroeconomic Policy After the Conservative Era. Cambridge, UK: Cambridge University Press, 143-71.

Clemente, J., A. Montañés y M. Reyes (1998): “Testing for a Unit Root in Variables with a Double Change in the Mean”. Economic Letters, 59, 2, 175-182.

Cline, W. (1997): Trade and Income Distribution. Washington: Institute for International Economics.

Edwards, S. (1993): "Openess, Trade Liberalization, and Growth in Developing Countries”. Journal of Economic Literature XXXI (3), 1358-1393.

-. (1998): "Openness, Productivity and Growth: What Do We Really Know? Economic Journal 108 (march), 383-398.

Esfahani, H.S. (1991): "Exports, Imports, and Growth in Semi-Industrialized Countries". Journal of Development Economics 35(1), 93-116.

Feder, G. (1983): "On Exports and Economic Growth: What Do We Really Know?". Journal of Development Economics 12 (1/2), 59-73.

Frankel, J. y D. Romer (1999):" Does Trade Cause Growth?". American Economic Review 89 (3), 379-393.

Harrison, A. (1996): "Openness and Growth: A Time-Series, Cross-Country Analysis for Developing Countries". Journal of Development Economics 48, 419-447.

Helpman, E. (2016): "Globalization and Wage Inequality”. NBER Working Paper $n^{\circ} 22944$.

Krugman, P. (1995): "Growing World Trade: Causes and Consequences”. Brooking Papers on Economic Activity, 1 (spring), 327-377.

--(2008): "Trade and Wages Reconsidered". Broking Papers on Economic Activity (spring), 103-154.

Lawrence, R. Z. (1996): Single World, Divided Nations? International Trade and OECD Labor Markets, Brookings. 
Lee, J.W. (1993): "International Trade, Distortions, and Long-Run Economic Growth”. International Monetary Found Sataff Papers 40 (2), 299-328.

Nelson, C. and C. Plosser, (1982): "Trends and Random Walks in Macroeconomic Time Series: Some Evidence and Implications," Journal of Monetary Economics 10, 139-162.

Onaran, O (2008): The Effects of Globalization on Employment, Wages and Wage Share in Austria". Working Paper Wien University.

- (2009). Wage Share, Globalization and Crisis: The Case of the Manufacturing Industry in Korea, Mexico and Turkey. International Review of Applied Economics, 23 (2), 113-134.

Papell, David H., Christian J. Murray, and Hala Ghiblawi. "The Structure of Unemployment." The Review of Economics and Statistics 82, no. 2 (2000), 309-15.

Pappell, D.H. y R. Prodan (2011): "The Statistical Behavior of GDP after Financial Crises and Severe Recessions”, en http://ssrn.com/abstract = 1933988.

Perron, P. (1989): "The Great Crash, the Oil Price Shock, and the Unit Root Hypothesis", Econometrica, 57, 1361-1401.

(1990): Testing for a Unit Root in a Time Series with a Changing Mean. Journal of Business \& Economic Statistics, 8(2), 153-162.

Perron, P., \& Vogelsang, T. (1992): "Testing for a Unit Root in a Time Series with a Changing Mean: Corrections and Extensions”, Journal of Business \& Economic Statistics, 10(4), 467-470.

Rodriguez, F; y D. Rodrik (2001): "Trade Policy and Economic Growth: A skeptic's Guide to the Cross-National Evidence". NBER Macroeconomics Annual 2000 (15), $261-338$.

Sachs, J.; A. Warner (1995): "Economic Reform and the Process of Global Integration". Brooking Papers on Economic Activity 1995(1), 1-118.

Stockhammer, E., E. Hein, y L. Grafl (2011): "Globalization and the Effects of Changes in the Functional Income Distribution in Aggregate Demand in Germany". International review of Applied Economics, 25 (1), 1-24.

Stockhammer, E. y Ö. Onaran (2012): "Wage-led Growth: Theory, Evidence, Policy". Working Paper Series of PERI (300), 1-32.

Stockhammer, E., Ö. Onaran, y S. Ederer (2009): "Functional Income Distribution and Aggregate Demand in the Euro Area”. Cambridge Journal of Economics, 33 (1), 139-59.

Wacziarg, R. (1998): "Measuring the Dynamic Gains from Trade". World Bank Working Paper 2001.

Zivot, E., and D. Andrews, (1992): "Further Evidence on the Great Crash, the Oil-Price Shock, and the Unit Root Hypothesis, Journal of Business and Economic Statistics, 10, 251-270. 